\title{
Hypothesis
}

\section{Why Do Some Reports Claim that the Number of COVID-19 Hospitalized Smokers is Smaller than Expected?}

\author{
Bevelacqua J. J. ${ }^{1 \oplus}$, Masoompour S. M. ${ }^{2}$, Mortazavi S. A. R. ${ }^{3}$, \\ Mortazavi S. M. J.4,5*๑
}

\begin{abstract}
Researchers believe that smoking might increase the severity of infections caused by SARS-CoV-2 by altering the viability and integrity of the BBB, while promoting the expression of ACE2 in endothelial cells, glia, and neurons. Moreover, as smoking increases the blood circulatory level of VWF and decreases the levels of thrombomodulin, it can lead to dysregulated blood homeostasis and hence, increased risk of thrombosis which significantly increases the risk of stroke and cardiovascular disorders. However, despite current controversies and remarkable study limitations, a recent systematic review and meta-analysis claims that the findings of 18 recent COVID-19 clinical and epidemiological studies show that smokers were statistically less likely to be hospitalized. The authors state that their observations might be due to the effects of nicotine. We believe that the lower number of hospitalized smokers than expected, if confirmed by further studies, can be due to the higher concentration of deposited energy of alpha particles emitted from radionuclides such as Po-210 in cigarette tobacco. The antiinflammatory effects of localized radiation energy deposition of alpha particles as well as the effect of low doses of radiation on reducing the risk of thrombosis, are possibly involved in the lower number of hospitalized smokers than expected.
\end{abstract}

Citation: Bevelacqua JJ, Masoompour SM, Mortazavi SAR, Mortazavi SMJ. Why Do Some Reports Claim that the Number of COVID-19 Hospitalized Smokers is Smaller than Expected? J Biomed Phys Eng. 2020;10(5):659-662. doi: 10.31661/jbpe.v0i0.2007-1144.

Keywords

COVID-19; Hospitalization; Smoking; Nicotine; Alpha Particles; LET

\section{Introduction}

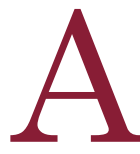

s of July 9, 2020, the global COVID-19 cases exceed 12 million and over 500,000 deaths have been reported. The US has surpassed 3 million COVID-19 cases; roughly a quarter of the world's total. Although a wide variety of potential therapeutic agents ranging from antivirals to steroids have been evaluated for the treatment of Covid-19, none have yet to be been fully effective. Substantial evidence shows that when the treatment is not fully effective and viral genome replicates, selective pressure leads the virus to rapid adaptation toward resistance "An increasing number of viral infections that impair host health are treated using antiviral drugs, typically targeting mechanisms of viral replication. If the treatment is robust and viral fitness is impaired sufficiently, no viral genomes will be successfully replicated, but if treatment is not as effective and some genomes replicate, selective pressure may result in rapid adaptation toward resistance".
${ }^{1} \mathrm{PhD}$, Bevelacqua

Resources, Richland,

Washington 99352,

United States

${ }^{2} \mathrm{MD}$, Non-Communica-

ble Diseases Research

Center, Shiraz University

of Medical Sciences,

Shiraz, Iran

${ }^{3} \mathrm{MD}$, School of Medi-

cine, Shiraz University

of Medical Sciences,

Shiraz, Iran

${ }^{4} \mathrm{PhD}$, Department of

Medical Physics and

Engineering, School of

Medicine, Shiraz Univer-

sity of Medical Sciences,

Shiraz, Iran

${ }^{5} \mathrm{PhD}$, lonizing and

Non-Ionizing Radiation

Protection Research

Center, Paramedical

School, Shiraz University

of Medical Sciences,

Shiraz, Iran

*Corresponding author:

S. M. J. Mortazavi

Department of Medical

Physics and Engineering,

School of Medicine, Shi-

raz University of Medical

Sciences, Shiraz, Iran

E-mail: mortazavismj@

gmail.com

Received: 17 July 2020

Accepted: 15 August 2020 
Low Dose Radiation as a Treatment for COVID-19

Successful use of ionizing radiation for the treatment of pneumonia dates back to the first half of the $20^{\text {th }}$ century. Given this consideration, a single dose of radiation instead of antiviral drugs has been recently suggested by different groups of scientists including Ghadimi-Moghadam et al. who first introduced this treatment method for severe pneumonia associated with COVID-19 [1, 2]. It is worth noting that radiation, at low doses, cannot exert a significant selective pressure on SARS-CoV-2, and hence does not lead the virus to "accelerated evolution" through mutations [3].

\section{The Challenging Issue of the Smok- ing}

A study conducted in Saudi Arabia in 2016 showed that smoking was significantly associated with MERS-CoV disease [4]. Regarding SARS-CoV-2, the virus causing coronavirus disease 2019 (COVID-19), a growing body of evidence confirms the detrimental effect of tobacco on blood-brain barrier (BBB) integrity. Given this consideration, researchers have speculated that smoking might increase the severity of infections caused by SARSCoV-2 by altering the viability and integrity of the $\mathrm{BBB}$, while promoting the expression of ACE2 in endothelial cells, glia, and neurons [5]. It is worth noting that SARS-CoV-2 uses the angiotensin-converting enzyme 2 (ACE2) as a cell receptor to invade human cells [6]. Moreover, as smoking increases the blood circulatory level of VWF and decreases the levels of thrombomodulin, it can lead to dysregulated blood homeostasis and hence, increased risk of thrombosis which significantly increases the risk of stroke and cardiovascular disorders [5].

However, a systematic review and metaanalysis published recently claims that the findings of 18 recent COVID-19 clinical and epidemiological studies show that smokers were statistically less likely to be hospitalized
$(\mathrm{OR}=0.18,95 \% \mathrm{CI}: 0.14-0.23, \mathrm{p}<0.01)$ [7]. Thus, the authors state: "The number of hospitalized smokers was smaller than expected based on the smoking prevalence in the different countries. The meta-analysis results obtained in China, US and Italy indicated that smoking habit lowers the likelihood of being hospitalized by COVID-19". The authors believe that their observations might be due to the effects of nicotine "We suspect that nicotine could be contribute to an amelioration of the cytokine storm and severe related inflammatory response through the $\alpha 7 n A C h R$-mediated cholinergic anti-inflammatory pathway during patient's aggravation". It is worth noting that the authors have already confirmed that their systematic review and presented meta-analyses had some limitations.

In contrast with the findings of the previous systematic review and meta-analysis, another systematic review calculates that the smokers were 1.4 times more likely $(\mathrm{RR}=1.4,95 \%$ CI: $0.98-2.00)$ to show severe symptoms of COVID-19 and about 2.4 times more likely to need intensive care (being admitted to an ICU), mechanical ventilation or die compared to non-smokers ( $\mathrm{RR}=2.4,95 \% \mathrm{CI}$ : $1.43-4.04)$. Despite their limited available data, and although the data were not adjusted for other factors that may influence the progression of the disease, the authors conclude that smoking is most likely linked to the negative progression and adverse outcomes in COVID-19 patients [8].

It should be noted that a systematic review and meta-analysis of data from more than 7 million participants, 99 cohort studies and over 50000 incident cases of lung cancer, previously showed the 7-fold higher RRs of lung cancer associated with smoking [9] that was considerably smaller than the Million Women's Study [10] and the British Doctors Study [11] that found the 20-fold increased risks. Given this consideration, smoking should be considered as one of the key causes of death and illness. However, regarding the 
COVID-19 and Smoking: Alpha Emitters vs Nicotine

lower number of hospitalized smokers than expected, if confirmed by further studies, we believe that the higher concentration of deposited energy of alpha particles are involved in this phenomenon. It should be noted that in 1960s, Po-210, a radionuclide that emits highLET alpha radiation, was being considered as a tobacco smoke constituent [12-14]. Despite efforts of tobacco manufacturers removing this radioactive substance from their products has been failed, so far. The highly localized energy deposition of alpha particles can cause severe damage to living cells. However, in this case, anti-inflammatory effects of localized radiation energy deposition of alpha particles as well reducing the risk of thrombosis by radiation can be involved in lower risk of hospitalization in smoker COVID-19 patients (Figure $1)$.

In summary, although we strongly believe that, at moderate or high levels of exposure, alpha particles emitted from the Th-232 and U-238 natural decay series (e.g., Po-210) make this radiation type one of the most hazardous carcinogenic agents, the anti-inflammatory effects of localized radiation energy deposition of alpha particles as well as the effect of low doses of radiation on reducing the risk of thrombosis, might be involved in the lower number of hospitalized smokers than expected.

Researchers must be careful to separate the level of smoking that produces a net antiinflammatory benefit from the overall negative effects of smoking and their detrimental effects on the health of the individual. Lung degradation caused by the collective effects of alpha radiation and chemical toxins produced by smoking should also be addressed.

The individual effects from alpha radiation and toxic chemicals included in the smoke that contribute to lung detriment should also be noted. How these agents collectively impact inflammation should be carefully studied. It is difficult to justify the importance of a single agent (i.e., nicotine) without evaluating the entire spectrum of agents present in the smoke.

Finally, the condition of the lungs before the COVID-19 infection and degree of smoking by the infected individual must be carefully evaluated. There is likely an optimum level of smoking that promotes an anti-inflammatory

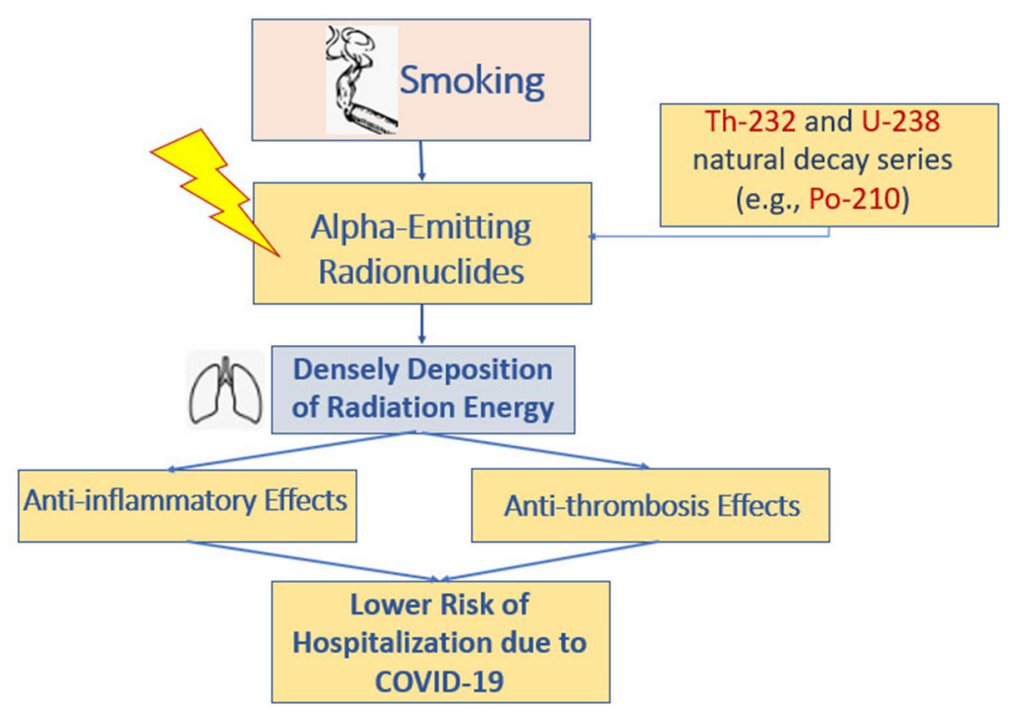

Figure 1: Both anti-inflammatory and anti-thrombosis effects of localized radiation energy deposition of alpha particles can be involved in lower risk of hospitalization in smoker COVID-19 patients. 
effect. Beyond that level, the detriment will exceed the benefit. In addition, there is likely an initial lung level of health required before the effects on smoking could be realized. Excessive smoking damages the lung to an extent that its possible benefits could not be realized.

\section{Conflict of Interest}

None

\section{References}

1. Ghadimi-Moghadam A, Haghani M, Bevelacqua JJ, Jafarzadeh A, Kaveh-Ahangar A, Mortazavi SMJ, Ghadimi-Moghadam A, Mortazavi SAR. COVID-19 tragic pandemic: concerns over unintentional "directed accelerated evolution" of novel Coronavirus (SARS-CoV-2) and introducing a modified treatment method for ARDS. J Biomed Phys Eng. 2020;10(2):241-6. doi: 10.31661/jbpe.v0i0.20031085. PubMed PMID: 32337192. PubMed PMCID: PMC7166223.

2. Mehdizadeh AR, Bevelacqua JJ, Mortazavi SAR, Mortazavi SMJ. COVID-19: Introducing Low Dose Radiation as an Effective Treatment for Pneumonia that Shouldn't Induce Selective Pressure and New Mutations. J Biomed Phys Eng. 2020;10(3):247-

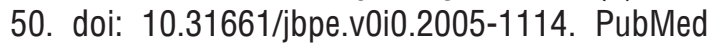
PMID: 32637368. PubMed PMCID: PMC7321390.

3. Mortazavi SMJ, Kefayat A, Cai J. Low dose radiation as a treatment for COVID-19 pneumonia: a threat or real opportunity? Med Phys. 2020. doi: 10.1002/mp.14367. PubMed PMID: 32619276. PubMed PMCID: PMC7362107.

4. Alraddadi BM, Watson JT, Almarashi A, Abedi GR, Turkistani A, Sadran M, Housa A, Almazroa MA, Alraihan N, Banjar A, Albalawi E. Risk factors for primary Middle East respiratory syndrome coronavirus illness in humans, Saudi Arabia, 2014. Emerg Infect Dis. 2016;22(1):49-55. doi: 10.3201/ eid2201.151340. PubMed PMID: 26692185. PubMed PMCID: PMC4696714.

5. Archie SR, Cucullo L. Cerebrovascular and Neurological Dysfunction under the Threat of $\mathrm{CO}$ VID-19: Is There a Comorbid Role for Smoking and Vaping? Int J Mol Sci. 2020;21(11):3916. doi: 10.3390/ijms21113916. PubMed PMID: 32486196. PubMed PMCID: PMC7312781.
6. Li MY, Li L, Zhang Y, Wang XS. Expression of the SARS-CoV-2 cell receptor gene ACE2 in a wide variety of human tissues. Infect Dis Poverty. 2020;9(1):45. doi: 10.1186/s40249-020-00662-x. PubMed PMID: 32345362 . PubMed PMCID: PMC7186534.

7. Gonzalez-Rubio J, Navarro-Lopez C, Lopez-Najera E, Lopez-Najera A, Jiménez-Díaz L, Navarro-López JD, Nájera A. What is Happening with Smokers and COVID-19? A Systematic Review and a Meta-Analysis. Preprints. 2020. doi: 10.20944/preprints202004.0540.v1.

8. Vardavas CI, Nikitara K. COVID-19 and smoking: A systematic review of the evidence. Tob Induc Dis 2020;18:20. doi: 10.18332/tid/119324. PubMed PMID: 32206052. PubMed PMCID: PMC7083240.

9. O'Keeffe LM, Taylor G, Huxley RR, Mitchell P, Woodward M, Peters SA. Smoking as a risk factor for lung cancer in women and men: a systematic review and meta-analysis. $B M J$ open. 2018;8(10):e021611. doi: 10.1136/bmjopen-2018-021611. PubMed PMID: 30287668. PubMed PMCID: PMC6194454.

10. Pirie K, Peto R, Reeves GK, Green J, Beral V, Million Women Study Collaborators. The 21st century hazards of smoking and benefits of stopping: a prospective study of one million women in the UK. The Lancet. 2013;381(9861):133-41. doi: 10.1016/S0140-6736(12)61720-6. PubMed PMID: 23107252. PubMed PMCID: PMC3547248.

11. Doll R, Peto R, Boreham J, Sutherland I. Mortality in relation to smoking: 50 years' observations on male British doctors. Bmj. 2004;328(7455):1519. doi: $\quad 10.1136 / \mathrm{bmj} .38142 .554479 . \mathrm{AE}$. PubMed PMID: 15213107. PubMed PMCID: PMC437139.

12. Muggli ME, Ebbert JO, Robertson C, Hurt RD. Waking a sleeping giant: the tobacco industry's response to the polonium-210 issue. Am J Public Health. 2008:98(9):1643-50. doi: 10.2105/ AJPH.2007.130963. PubMed PMID: 18633078. PubMed PMCID: PMC2509609.

13. Radford EP, Hunt VR. Polonium-210: a volatile radioelement in cigarettes. Science. 1964;143(3603):247-9. doi: 10.1126/science.143.3603.247. PubMed PMID: 14078362.

14. Winters TH, Di Franza JR. Radioactivity in cigarette smoking. N Engl J Med. 1982;306(6):364-5. doi: 10.1056/NEJM198202113060612. 